\title{
Using Kiefer Density Matrix for Time Flow Analysis and How This Links to a Proof of Production of Planck Mass BHs during Inflation and Their Resulting Breakup, Leading to a DE Candidate
}

\author{
Andrew Beckwith \\ Physics Department, College of Physics, Chongqing University, Chongqing, China \\ Email: rwill9955b@gmai.com
}

How to cite this paper: Beckwith, A. (2021) Using Kiefer Density Matrix for Time Flow Analysis and How This Links to a Proof of Production of Planck Mass BHs during Inflation and Their Resulting Breakup, Leading to a DE Candidate. Journal of High Energy Physics, Gravitation and Cosmology, 7, 1005-1018.

https://doi.org/10.4236/jhepgc.2021.73059

Received: June 1, 2021

Accepted: July 3, 2021

Published: July 6, 2021

Copyright (c) 2021 by author(s) and Scientific Research Publishing Inc. This work is licensed under the Creative Commons Attribution International License (CC BY 4.0).

http://creativecommons.org/licenses/by/4.0/

\begin{abstract}
We are using the book "Towards Quantum Gravity" with an article by Claus Kiefer as to a quantum gravity interpretation of the density matrix in the early universe. The density matrix we are using is a one loop approximation, with inflaton value and potential terms, like V (phi) using the Padmanabhan values one can expect if the scale factor is a $\sim$ a (Initial) times $t \wedge$ gamma. In doing so, we identify two time steps and presume a very small initial time step candidates initial time values which are from a polynomial for time values. A gravity wave analysis concludes our article with inflaton decay, which is finally linked to BHs. And then finally we show using work done by Hawking, et al. how this may give us Planck Sized Black Holes, in the onset of Inflation, with resulting consequences so outlined. A vastly simplified proof of $\mathrm{BH}$ masses of Planck mass is presented which ties in directly with issues of the mass of the inflaton initially generated by the $2^{\text {nd }}$ derivative of the effective potential V (phi) at a time $\mathrm{t} \sim 4$ times Planck time. And we include at the close questions as to $\mathrm{DE}$, and data sets which may give credence to speculation as to different time flow rates at the start and then the conclusion later on, of expansion of our universe. The DE would be created by the breakup of the black holes due to a mechanism brought up by Dr. Freeze in 2012, and we also are using the future works section 8 to define the contours of our DE model which builds upon quite directly the sequence of material from pages 1 to 9 which are cited as to making connection between early universe conditions and the ideas of primordial DE models.
\end{abstract}




\section{Keywords}

Minimum Scale Factor, Cosmological Constant, Plank Mass BH, DE

\section{Introduction}

What we are doing is to examine several different items of research inquiry

1) The Kieffer Density function is introduced;

2) We next then ask how to obtain inflaton mass;

3) We then next establish a probability density mass locator;

4) Applying our ideas above to determining likelihood of Plank sized black holes;

5) Using the existence of relic Black holes, if the break up exists defacto to outline DE initially;

6) And then to see if this is linkable eventually to Dr. Bakers different rates of time flow theory for the early universe.

Our initial goal is to obtain, via a Kieffer Density function candidate minimum time steps which will be for the purpose of giving input into an uncertainty principle of the form [1] [2] [3],

$$
\Delta E \Delta t \approx 4 \hbar
$$

Whereas our candidate [4] for a density matrix uses the following treatment of the Potential term used again and again in this document.

$$
H^{2}=V_{0} \exp \left(-\sqrt{\frac{16 \pi G}{v}} \phi\right)=V_{0} \cdot\left(\sqrt{\frac{8 \pi G V_{0}}{v \cdot(3 v-1)}} \cdot t\right)^{\frac{1}{2} \cdot \sqrt{\frac{v}{\pi G}}-4 \sqrt{\frac{\pi G}{v}}}
$$

where [5] [6] in turn is referenced directly to having use of the following:

$$
\begin{aligned}
& a(t)=a_{\text {initial }} t^{v} \\
& \Rightarrow \phi=\ln \left(\sqrt{\frac{8 \pi G V_{0}}{v \cdot(3 v-1)}} \cdot t\right)^{\sqrt{\frac{v}{16 \pi G}}} \\
& \Rightarrow \dot{\phi}=\sqrt{\frac{v}{4 \pi G} \cdot t^{-1}} \\
& \Rightarrow \frac{H^{2}}{\dot{\phi}} \approx \sqrt{\frac{4 \pi G}{v}} \cdot t \cdot T^{4} \cdot \frac{(1.66)^{2} \cdot g_{*}}{m_{P}^{2}} \approx 10^{-5}
\end{aligned}
$$

In doing all of this we are making full use of the following from [4] due to a one loop approximation,

$$
\rho(\phi, \phi) \approx\left\{\exp \left(\frac{ \pm 3 M_{P}^{4}}{8 \cdot V(\phi)}\right)\right\} \cdot \phi^{-\tilde{Z}-2}
$$

Which after we isolate out $\Delta t$ makes use of Equation (1), which is derived as given in [1] [2] [3]. 


$$
\begin{gathered}
\Delta t \geq \frac{\hbar}{\Delta E}+\gamma t_{P}^{2} \frac{\Delta E}{\hbar} \Rightarrow(\Delta E)^{2}-\frac{\hbar \Delta t}{\gamma t_{P}^{2}}(\Delta E)^{1}+\frac{\hbar^{2}}{\gamma t_{P}^{2}}=0 \\
\Rightarrow \Delta E=\frac{\hbar \Delta t}{2 \gamma t_{P}^{2}} \cdot\left(1+\sqrt{\left.1-\frac{4 \hbar^{2}}{\gamma t_{P}^{2} \cdot\left(\frac{\hbar \Delta t}{2 \gamma t_{P}^{2}}\right)^{2}}\right)}\right)=\frac{\hbar \Delta t}{2 \gamma t_{P}^{2}} \cdot\left(1 \pm \sqrt{1-\frac{16 \hbar^{2} \gamma t_{P}^{2}}{(\hbar \Delta t)^{2}}}\right) \\
\Delta E \approx \frac{\hbar \Delta t}{2 \gamma t_{P}^{2}} \cdot\left(1 \pm\left(1-\frac{8 \hbar^{2} \gamma t_{P}^{2}}{(\hbar \Delta t)^{2}}\right)\right) \\
\Rightarrow \Delta E \approx \text { either } \frac{\hbar \Delta t}{2 \gamma t_{P}^{2}} \cdot \frac{8 \hbar^{2} \gamma t_{P}^{2}}{(\hbar \Delta t)^{2}}, \text { or } \frac{\hbar \Delta t}{2 \gamma t_{P}^{2}} \cdot\left(2-\frac{8 \hbar^{2} \gamma t_{P}^{2}}{(\hbar \Delta t)^{2}}\right) \\
\Delta E \approx \frac{\hbar \Delta t}{2 \gamma t_{P}^{2}} \cdot \frac{8 \hbar^{2} \gamma t_{P}^{2}}{(\hbar \Delta t)^{2}} \equiv \frac{4 \hbar}{\Delta t}
\end{gathered}
$$

We will be applying Equation (4) to obtain $\Delta t$, and then from this step applying Equation (1) to say foundational import issues of time flow in the beginning, as it affects initial energy values and from there obtain some matters of observational import in GW astronomy.

\section{Understanding the Import of Equations (2)-(4) for $\Delta t$}

Our assumption is that time, $t$, which becomes $\Delta t$ is extremely small. Hence without loss of generality we write, if as an example, $\tilde{Z} \approx 2$.

And we simplify time dependence by setting $v=\frac{3 \pi}{\sqrt{2}}$ in Equations (2)-(4).

Then, without loss of generality, if we observe this, and set $\Theta$ as a probability density value of Equation (4), we then have:

$$
\left(\frac{1}{4} \cdot \exp \left( \pm \frac{3}{8} \cdot \frac{M_{P}^{4}}{V_{0}}\right)\right) \cdot\left(1-4 \cdot\left(\sqrt{\frac{8 \pi V_{0}}{\left(\frac{3 \pi}{\sqrt{2}}\right) \cdot\left(\frac{9 \pi}{\sqrt{2}}-1\right)}}\right) \cdot \Delta t\right) \approx \Theta
$$

If so, then we have a minimum time step of the form:

$$
\Delta t \approx 4\left(\sqrt{\left.\frac{\left(\frac{3 \pi}{\sqrt{2}}\right) \cdot\left(\frac{9 \pi}{\sqrt{2}}-1\right)}{8 \pi V_{0}}\right)} \cdot\left(1-4 \Theta \exp \left( \pm \frac{3}{8} \cdot \frac{M_{P}^{4}}{V_{0}}\right)\right)\right.
$$

\section{Interpreting Equation (9) in Terms of the Affects It Has on Equation (1)}

We have to consider what $\Theta$ may or may not be. The core of the derivation of Equation (4) in [4] due to [7] is dependent on having the following, namely,

Quote: 
The quantum gravitational scale of inflation is calculated by finding a sharp probability peak in the distribution function of chaotic inflationary cosmologies driven by a scalar field with large negative constant $\Xi$ of nonminimal interaction. In the case of the no-boundary state of the universe this peak corresponds to the eternal inflation, while for the tunnelling quantum state it generates a standard inflationary scenario. The sub-Planckian parameters of this peak (the mean value of the corresponding Hubble constant $\mathrm{H} \cong 10^{-5} \mathrm{mp}$, its quantum width $\Delta \mathrm{H} / \mathrm{H}$ $\cong 10^{-5}$ and the number of inflationary e-foldings $\mathrm{N} \cong 60$ ) are found to be in good correspondence with the observational status of inflation theory, provided the coupling constants of the theory are constrained by a condition which is likely to be enforced by the (quasi) supersymmetric nature of the sub-Planckian particle physics model.

End of quote:

Notice here that this is akin to making use of Equation (3.4) of [7] so that we have restrictions on particle manufacturing for the theory. This is in line with:

$$
\Delta E \approx \hbar \cdot\left(\sqrt{\frac{8 \pi V_{0}}{\left(\frac{3 \pi}{\sqrt{2}}\right) \cdot\left(\frac{9 \pi}{\sqrt{2}}-1\right)}}\right) \cdot\left(1+4 \Theta \exp \left( \pm \frac{3}{8} \cdot \frac{M_{P}^{4}}{V_{0}}\right)\right)
$$

The consequences for frequency of signals are as follows. First, we make an estimation as to the width of the wavefront of a DeBroglie wave, which may be a consequence of a signal, as well as the position of the phenomenon of generation of say Gravitons. In doing so we wish to refer to the following as motivation in order to link this to graviton mass and other such concerning heavy gravity, In [8] we have the following, as to how to obtain the mass of an inflaton, namely use, if,

$$
\begin{aligned}
& m^{2}=\left.\frac{\mathrm{d}^{2} V}{\mathrm{~d} \phi^{2}}\right|_{\phi=0, t=3.9776 \cdot t_{P}}=\frac{16 \pi}{3 \pi / \sqrt{2}} \\
& \Rightarrow m=2.74635619187
\end{aligned}
$$

IMO the inflaton mass is 2.746356 times Planck Mass, and this is a starting value of inflaton mass at $t=3.9776$ planck time.

Having this value of inflaton mass should be compared with the value of energy density as given by:

$$
\rho \approx \frac{\dot{\phi}^{2}}{2}+V(\phi) \equiv \frac{\gamma}{8 \pi G} \cdot t^{2}+V_{0} \cdot\left\{\sqrt{\frac{8 \pi G V_{0}}{\gamma(3 \gamma-1)}} \cdot t\right\}^{\sqrt{\frac{\gamma}{4 \pi G}}-\sqrt{\frac{8 \pi G}{\gamma}}}
$$

Using the coefficient of the scale factor chosen, this above becomes simplified to become, if,

$$
V_{0} \approx M_{P}^{4} \underset{\hbar=\ell_{P}=t_{P}=k_{B}=G=1}{\longrightarrow} 1
$$

This is a way to obtain the following value for density as can be seen below:

$$
\rho=\frac{3 \sqrt{2}}{8} \cdot\left(\frac{3.997+\varpi}{t_{P} \underset{\hbar=\ell_{P}=M_{P}=k_{B}=G=1}{\longrightarrow} 1}\right)^{\frac{3 \pi}{\sqrt{2}}}+\left(V_{0} \approx M_{P}^{4} \underset{\hbar=\ell_{P}=t_{P}=k_{B}=G=1}{\longrightarrow} 1\right)
$$


We then can look at how this will be larger than Planck energy where our starting point will be if we set $\varpi$ close to zero, and then have, due to how close the time is to Planck time, a situation for which we are looking at nearly Planck length, cubed as a starting volume, then we have initially, having a near Planck sized initial volume, we have then,

$$
1+\left(\frac{3 \sqrt{2}}{8}\right) \cdot(3.997+\varpi)^{\frac{3 \pi}{\sqrt{2}}} \approx \sqrt{\frac{8 \pi}{\left(\frac{3 \pi}{\sqrt{2}}\right) \cdot\left(\frac{9 \pi}{\sqrt{2}}-1\right)}} \cdot\left(1+4 \Theta \cdot \exp \left( \pm \frac{3}{8}\right)\right)
$$

Then up to an initial round off error, in the beginning, we can have:

$$
\begin{aligned}
& \Theta \approx \frac{1}{4} \cdot\left[\exp \left( \pm \frac{3}{8}\right)\right] \cdot\{\} \\
& \{\}=\left\{-1+\sqrt{\frac{\left(\frac{3 \pi}{\sqrt{2}}\right) \cdot\left(\frac{9 \pi}{\sqrt{2}}-1\right)}{8 \pi}} \cdot\left[1+\left(\frac{3 \sqrt{2}}{8}\right) \cdot(3.997+\varpi)^{\frac{3 \pi}{\sqrt{2}}}\right]\right\}
\end{aligned}
$$

$\Theta$ is in the initial setting a way to bring up what $\Theta$ is as a probability density value, and this is the point to remember.

In quantum mechanics, probability amplitude is a complex number used in describing the behaviour of systems. The modulus squared of this quantity represents a probability density. Note that the Interpretation of values of a wave function as the probability amplitude is a pillar of the Copenhagen interpretation of quantum mechanics.

We are then reproducing in this method the idea of [9] namely that the following is true.

Probability Density of Particles: The probability density (or probably distribution) is given by taking the square of the absolute value of the wave function. It gives us the likelihood of finding an electron (or some other system) at some given point in space.

\section{Making Use of the Idea of a Nonzero Probability Density of "Masses" of Some Particle within the Copenhagen Interpretation of Quantum Mechanics, in the Vicinity of the Big Bang}

Here what we are going to say, is that due to a fluctuation in time, given by Equation (9), that the probability density of finding, say traces of the inflaton, as given in Equation (11) will be nonzero, and varying in ways which could be experimentally tested. Given a mass, $\mathrm{m}$, as in Equation (11) could be interpreted as being of value of effective space-time mass $>1$ Planck mass, and if these are broken apart, in the matter of [10] for black holes, we could have, with the evolution of time a template for investigating the applicability of graviton masses being generated by black holes being broken up in the vicinity of a quantum bounce, with each quantum generated. 


$$
m_{g}=\frac{\hbar \sqrt{\Lambda}}{c}
$$

Whereas we have a thermality relationship which may be useful for analysis of the form given by

$$
\frac{H^{2}}{\dot{\phi}} \approx 10^{-5}
$$

And also the use of the following at the start of inflation, as given in [11] and [12] as well as the ideas given by Uptal Sarkar in [13] and [14]. We will also be examining if our construction will allow for the development of spin-off of ideas given by Equation (13) and (14),

$$
H=1.66 \sqrt{g_{*}} \cdot \frac{T_{\text {temp }}^{2}}{m_{P}}
$$

The term $g_{*}$ can refer to the initial degrees of freedom and can go as high as 110 , whereas Equation (13) is a bound in the amount of inhomogenity. Whereas our future research objective is to find a way to allow the idea of a nonzero probability density, to ascertain different values of say graviton production if we make use of having from Freeze [10] of a mechanism of breaking up initially speaking black holes, due to the following criteria as given by Freeze,

$$
\rho_{\text {BH breakup density }}=\frac{M_{P}^{4}}{32 \pi} \cdot\left(\frac{M_{P}^{4}}{m^{4}}\right) \cdot \frac{1}{\left|1+3 \omega_{Q}\right|}
$$

This breakup of black holes by the physics so outlined may give us a way to ascertain if the following entropy, initially is verifiable experimentally, whereas we wish to examine in full ideas given in the series of multiple references [15]-[25], starting with verification of

$$
S \sim 3 \cdot\left[1.66 \sqrt{g_{*}}\right]^{2} T^{3}
$$

\section{Conclusion: Examining the Origins of Density Fluctuations, as Given in This Formalism}

In doing this final part of the inquiry we are making use of [26] and in doing so we are looking at the following on page 299 of [26], namely,

$$
\widehat{\hat{a}} \cdot \ddot{\phi}+3 \cdot \widehat{\hat{a}} \cdot H \cdot \dot{\phi}+\frac{\mathrm{d} V}{\mathrm{~d} \phi}(\phi)+(\mathfrak{I} / \dot{\phi})=0
$$

Then, if one has a time $\sim 10$ times Planck time, one has:

$$
\begin{aligned}
& \mathfrak{I} \approx \frac{2}{\left(10 \cdot t_{P}\right)}-\left(3 \cdot\left(\frac{3 \pi}{\sqrt{2}}-1\right)\right) \cdot\left(\frac{\hat{\widehat{a}}=15 / 2}{\left(10 \cdot t_{P}\right)^{3}}\right) \\
& \equiv \underset{t_{P} \rightarrow 1}{\longrightarrow} \frac{2}{10}-\left(3 \cdot\left(\frac{3 \pi}{\sqrt{2}}-1\right)\right) \cdot\left(\frac{15 / 2}{10^{3}}\right)>0
\end{aligned}
$$

$\mathfrak{I}$ in this situation is a way of representing the energy drain created by the radiation of "particles" which couple to the field given by $\phi$, i.e. this is the big 
news, namely that as early as a time value past the onset of the big bang, measured as about 10 times Planck time, i.e. roughly $10^{-42}$ seconds, we have an energy drain caused by something which is generated by the inflaton "mass" likely breaking up into radiated "particles" which are generated as the inflaton mass is created by Equation (11) above.

Furthermore, making use of Equation $\Theta$ we have a way of representing the likelyhood of particles being in existence in a particular regime of space-time. Taking into account having time $t$ in between 4 to 10 times Planck time, we can state that some version of the inflaton itself would exist in some order of magnitude of say 10 to 100 times Planck length, from the view point of [27], namely,

Quote:

We propose that there may be a substantial stochastic gravitational wave $(\mathrm{GW})$ background from particle origin, mainly from the gravitational three-body decay of the inflaton. The emitted gravitons could constitute a sizable contribution to dark radiation if the mass of inflaton is close to the Planck scale, which may be probed by future $\mathrm{CMB}$ experiments that have a sensitivity on the deviation of the effective number of neutrinos in the standard cosmology, $\delta \mathrm{N}_{\text {eff }} \sim 0.02-0.03$. We have also illustrated the spectrum of the radiated gravitational waves, in comparison to the current and future experiments, and found that GWs from particle origin could be the dominant contribution to the energy density at high-frequency domain, but beyond the sensitivity regions of various GW experiments in the near future.

End of quote:

The frequency range could be as high, initially as $10^{27} \mathrm{GHz}$ based upon the extremely small regime of space-time given in the initial creation of an inflaton which would be by 60 or more e folds [28] down graded in the present era down to about 1 to $10 \mathrm{GHz}$, from its initial configuration.

Keep in mind that the inflaton, as given in Equation (11) is over 2 times Planck mass, which is by Reference [27] more than enough to ensure that there is a GW signal from inflaton decay as initiated by the procedures given in [27]. The e fold values could be as low as 50 e folds, if we literally interpret [28], whereas what we are considering is a signal which may be so created. Keep in mind that the differential time flow, as specified in this problem for reasons we will investigate in future publications may make the difference between $10 \mathrm{GHz}$ and $1 \mathrm{GHz}$ relic inflaton decay generated $\mathrm{GW}$, i.e. the fact is, that this possibility cannot be dismissed out of hand until we obtain data sets. Also keep in mind what was brought up in [28].

Quote:

If future cosmological observations point to a particular inflaton potential for which our Universe is not generic under the canonical measure, that would shed light on even higher-scale physics. Such a circumstance would tell us that our Universe is tuned-on a non-generic trajectory-from the point of view of the classical measure. This would indicate the importance of intrinsically quantum gravitational processes or some ultimate theory of initial conditions. 
End of quote:

We hope that our discussion of a density functional as given in Equation (14) may be a step in this direction.

\section{Addendum to Conclusion. How This Procedure as Outlined Gives an Almost 100\% Chance of Planck Sized Early Universe Black Holes, and Resulting Signal Strength of GW}

On page 10 of [29], we have that there is a likelihood of pair production rate of Planck sized BHs Here $\varphi_{0}$ is the value of $\varphi$ in the initial Euclidean region. Thus the pair creation rate is given by, if $\varphi_{0}-:>0$, at $t \sim 4$ times Planck time of a value given by:

$$
\begin{aligned}
& \Gamma=\exp \left(-\frac{\pi}{V_{\text {eff }}}\right) \underset{\text { Planck regime }}{\longrightarrow} \exp \left(-\left.\frac{\pi}{8 \pi V_{0}}\right|_{t \approx 4 \times \text { Planck time }}\right) \\
& =\exp (-0.125) \approx 0.88249690258 \cong O(1)
\end{aligned}
$$

Equation (22) in comparison with the discussion at the bottom of page 10 of [29] signifies that there will be Plank sized Black holes, and at an astonishingly early age in the birth of the universe. More to the point, Equation (11) above, with its value of inflaton mass of about 2.74 times Planck mass indicates that within 4 times Planck time, since the start of inflation that there will be up to 2-3 Planck mass sized objects which may be obtainable. I.e. an extreme view of this Equation (11) result combined with Equation (22) indicates the likelyhood of 2 to 3 Plank sized black holes forming right in the process of inflation. The signal strength of resulting black holes, will come up as a future works project which will form the final chapter of this document.

\section{Generating Black Holes, and Their Possible Destruction, as Brought up by $\mathrm{K}$. Freeze, in [10] and Its Possible Relationship to DE?}

In [30] Claus Kiefer, outlined a claim that relic $\mathrm{BH}$ production could be a source for DM production. We will outline how instead this may be linked to relic DE and to do it as an extension of the arguments given in this document so far. What we are doing is to ascertain how the relic Black holes established by the Kieffer.

We will first start off with the redone calculation as to the Vacuum energy as given in [18] and how we rescale them to be in sync as to the observed experimental value for vacuum energy which is of the present era. This methodology is consistent with the Zero-point energy calculation, we start off with the following as given by [18]:

$$
\begin{aligned}
& \frac{1}{2} \cdot \sum_{i} \omega_{i} \equiv V(\text { volume }) \cdot \int_{0}^{\hat{\lambda}} \sqrt{k^{2}+m^{2}} \frac{k^{2} \mathrm{~d} k}{4 \pi^{2}} \approx \frac{\hat{\lambda}^{4}}{16 \pi^{2}} \\
& \underset{\hat{\lambda}=M_{\text {Planck }}}{\longrightarrow} \rho_{\text {boson }} \approx 2 \times 10^{71} \mathrm{GeV}^{4} \approx 10^{119} \cdot\left(\rho_{D E}=\frac{\Lambda}{8 \pi G}\right)
\end{aligned}
$$


In stating this we have to consider $\rho_{D E}=\frac{\Lambda}{8 \pi G} \approx \hbar \cdot \frac{(2 \pi)^{4}}{\lambda_{D E}^{4}}$, so then we have to consider a wavelength $\lambda_{D E} \approx 10^{30} \ell_{\text {Planck }}$ which is about $10^{30}$ times a Planck length radius of a space-time many times larger than that of the initial Planck radii regime, i.e.,

$$
\lambda_{D E} \approx 10^{30} \ell_{\text {Planck }}
$$

before the near singularity is to be solved. Then the existence of Equation (19) solves the problem.

In short, we have a much broader region of space-time for DE and this opens up a regime of space-time for analysis $10^{30}$ times larger than Planck length for examining our production of early universe black holes, and then by extension possibly the DE problem. If so, then if we assume that $1 / 1000$ of relic black hole mass may be converted to gravitons and that the Freeze break up of black holes as given in [10] may be correct, we get then the following

Look at when an object of mass $\mathrm{m}$, and radius $\mathrm{R}$ is pulled apart. From [10], page 154,

$$
m \approx \frac{8 \pi R^{3}(\text { radius }) \cdot \rho}{3}
$$

With this generalized to being for black holes being pulled apart when,

$$
\begin{aligned}
& m \approx-\left(\frac{4 \pi \rho}{3}\right) \cdot\left(1+3 \cdot \frac{P_{G}}{\rho_{G}}\right) \cdot \frac{8 m^{3}}{m_{P}^{6}} \\
& \Rightarrow \text { if } P_{G} \approx-\rho_{G} \\
& m(\text { black holes }) \geq 10^{5} m_{P} \text { break apart } \\
& \text { after } t \approx 10^{-27} \mathrm{sec}
\end{aligned}
$$

We then have, say that there are a large number of black holes of about $10^{5}$ Planck Mass at a time just after the end of the inflationary era, which are pulled apart, and if we then look at the formula of $1 / 1000$ of the mass of a black hole we are talking about a contribution of about $10^{2} \mathrm{~m}_{p}$ in black hole generation of a flux of gravitons after $10^{-27}$ seconds, i.e. for the regime from $10^{-33}$ seconds to $10^{-27}$ seconds, we get

$$
10^{2} m_{P}(\text { gravitons }) \Rightarrow 10^{67} \text { gravitons per } 10^{5} m_{P}(\text { black hole })
$$

\section{Future Project to Develop. Making DE Equivalent to a Sea of Initial Gravitons, via Break up of Initial Plank Mass to Slightly Larger BHs}

Here is a consideration to take into account before we commence on this analysis. If we have $10^{-26}$ seconds in evolving space-time acting as though it would for a time $10^{29}$ times larger due to gravitons traveling at nearly the speed of light, we would find that if we have a mass of say $10^{-65} \mathrm{~g}$ for the frame of reference of a graviton, we have an expansion of mass, effectively for a $10^{-65} \mathrm{~g}$ graviton increased in our frame of reference roughly $10^{30}$ times, which would leave $10^{-65} \mathrm{~g}$ 
transformed to $10^{-35} \mathrm{~g}$, so a $10^{-26}$ second interval would be expanded in relativity to be equivalent to 2.68 times $10^{3}$ seconds. In this case and way, the value of $10^{-26}$ seconds in the graviton mass frame of reference would then be for roughly $10^{3}$ seconds which would correspond roughly to 2.68 times $10^{3}$ seconds for 9 trillion kilometers.

Having made that clear, the bottom analysis can be made congruent although the algebra may still be way off.

The mass of the graviton, would still be inconceivably small, i.e. in the factored case still of the order of $10^{-35} \mathrm{~g}$, but $10^{30}$ times larger than in the offered rest frame mass of the graviton.

I await comments from referees as to how to clear this up, but the main datum still remains that we could have, in a demonstrable way a breakup of black holes, due to the mechanism discussed from Freeze et al. [10] with say 1/1000 of the mass of small black holes, contributing to gravitons, and the gravitons contributing to DE. If one wants an idea of how this could work in the later universe, one can observe the given mechanics of reacceleration of the universe due to the reference I will appeal to. Now for the story

Roughly put, one hydrogen atom is about 1.66 times $10^{-24}$ grams. The weight of a Massive graviton is about $10^{-65}$ grams hence we are talking about $10^{-22}$ grams, or about $10^{44}$ gravitons, with each graviton about $6 \times 10^{-32} \mathrm{eV} / \mathrm{c}^{2}$. After $10^{-27}$ seconds, the following in the set of equations given below are Equivalent, and that these together will lead to a cosmological Constant, $\Lambda$ of the sort which we will be able to refer to later

$$
\begin{gathered}
1 \text { graviton } \approx 10^{-65} \mathrm{~g} \\
M_{P B H} \sim 10^{15} \times\left(\frac{t=10^{-27} \mathrm{~s}}{10^{-23} \mathrm{~s}}\right) \cdot \mathrm{g}(\text { grams }) \approx 10^{11} \mathrm{~g} \approx 10^{16} \mathrm{~m}_{P}
\end{gathered}
$$

Assuming that gravitons contribute to the Dark Energy value will lead to us using the Karen Freeze model, with gravitons being released in the early universe by the breakup early universe black holes which have a maximum value of about $10^{11} \mathrm{~g}$, as opposed to the value of the Sun which has about $10^{33}$ grams, i.e. by making use of the following:

$$
\begin{aligned}
& \mathrm{DE}(\text { from broken black hole }) \sim 7 \times 10^{-30} \mathrm{~g} / \mathrm{cm}^{3} \approx 7 \times 10^{12} \mathrm{~g} /\left(10^{9} \mathrm{~km}\right)^{3} \\
& \text { Univese (black hole) } \sim 7 \times 10^{15} \mathrm{~g} /\left(10^{9} \mathrm{~km}\right)^{3} \approx 7 \times 10^{4} M_{P B H} /\left(10^{9} \mathrm{~km}\right)^{3}
\end{aligned}
$$

We claim that the above Equation (28c), will be able to yield a DE value $\Leftrightarrow$ $10^{80}$ gravitions in a region of space for which we have a "sphere" of radius $10^{9} \mathrm{~km}$ at $10^{-27}$ seconds as a way to support the existence of the mass of DE, i.e. about $10^{4}$ number of black holes given in Equation (28d) initially.

This needs to be confirmed in some way via data sets and it ties in directly with the initial motivation of this document which was [31] by Dr. Robert Baker to conform the likelihood of different rates of time flow in the early universe; The different initial time steps may well, with additional observations confirm 
[31] as well as the ideas given by Calmert et al. in [32] about the relative contribution of gravitons to black hole physics.

Alves et al. in [33] commenced to apply Gravitons as a replacement for DE in the later time of the reacceleration of the universe commencing one billion years ago. We are by way of contrast attempting to focus upon what would be a regime of the early universe space-time.

Our approach would link Gravitons to early universe DE. If, say the number of black holes broken up still leads to $10^{80}$ Gravitons, then comes the problem if or not the number of gravitons may be commensurate with forming a times arrow which would be commensurate with the [19] 't Hooft criteria as to initial conditions allowing for an orderly arrow of time hypothesis.

The next item would be, if gravitons are commensurate as to $\mathrm{DE}$, in line with our ideas different from [33] would be to investigate the signal from gravitons can be measured appropriately. That would entail doing analysis consistent with [34] as to the quadrupole analysis of GW strength, from perhaps millions of breaking up Black holes as well as the ideas of [35] among other things, for confirmation of optimal techniques.

Finally, although we are having in this situation a review of techniques, as to having a cosmological constant recovered along the lines of Problem 7, of page 265-266 of [36] which is entitles recovering (as on formula P33 of 266 of [36]) a way to ascertain a bare cosmological constant, which allows us to make a relationship to what is called zero point energy techniques. In short, with observational constraints utilized, a review of phenomenology so then that the procedure brought up on page 553, of [37] can in the detection of early universe GW, be linkable or give support to the following approximation for the effects of the cosmological constant, $\Lambda$ upon the solar system, which is dependent upon $\rho_{C} \approx \Lambda / 8 \pi$ so then we can if $\rho_{\text {solar system }} \approx 10^{-12} \mathrm{~g} / \mathrm{cm}^{3}$ then set

$$
\frac{\rho_{C}}{\rho_{\text {solar system }}} \approx 10^{-19}
$$

If this document passes review, it will be necessary to do significant data set modelling and analysis criteria. Equation (29) would represent a gold standard of instrumentation set points to work with. The derivation specified in [36] as to a bare cosmological constant should not be ignored and hopefully the t'Hooft criteria [19] should be attended to and confirmed in data set analysis to see if initial conditions for the arrow of time, Gravitons, DE, and the cosmological constant can be in experimental settings confirmed, vetted and made consistent with theoretical models.

\section{Acknowledgements}

This work is supported in part by National Nature Science Foundation of China grant No. 11375279. The author wishes to thank Dr. Christian Corda for encouragement as to this given research work being pursued in the first place, Dr. Fangyu $\mathrm{Li}$ and Dr. Wen Hao for alerting the author to the relevance of early 
universe conditions to GW analysis, and Dr. Robert Baker for proposing that time in the early universe may differ in its time flow characteristics from what we see today.

\section{Conflicts of Interest}

The author declares no conflicts of interest regarding the publication of this paper.

\section{References}

[1] Shalyt-Margolin, A.E. (2006) Deformed Density Matrix and 21 Quantum Entropy of the Black Hole. Entropy, 8, 31-43. https://doi.org/10.3390/e8010031

[2] Narlinkar, J.V. (1982) Quantum Fluctuations near the Classical Space-Time Singularity. Proceedings of the Workshop on Gravitation and Relativistic Astrophysics, Ahmedabhad, 18-20 January 1982, 135-151.

[3] Shalyt-Margolin, A.E. (2005) The Density Matrix Deformation in Physics of the Early Universe and Some of Its Implications. In: Quantum Cosmology Research Trends, Horizons in World Physics, Vol. 246, Chapter 2, Nova Science Publishers, Inc., Hauppauge, 49-91.

[4] Kiefer, C. (2000) Quantum Cosmology. In: Toward Quantum Gravity, Lecture Notes in Physics, Vol. 541, Springer-Verlag, New York, 158-187.

[5] Winitzki, S. (2009) Eternal Inflation. World Scientific Publishing Co., Singapore. https://doi.org/10.1142/6923

[6] Padmanabhan, T. (2006) An Invitation to Astrophysics. World Scientific Series in Astronomy and Astrophysics: Volume 8, World Press Scientific, Singapore.

https://doi.org/10.1142/6010

[7] Barvinsky, A.O. and Kamenschik, A.Yu. (1994) Quantum Scale of Inflation and Particle Physics of the Early Universe. Physics Letters B, 332, 270-276.

https://arxiv.org/pdf/gr-qc/9404062.pdf https://doi.org/10.1016/0370-2693(94)91253-X

[8] Katlesh, R. (2007) M String Theory and Anthropic Reasoning. In: Carr, B., Ed., Universe or Multiverse, Cambridge University Press, New York, 191-210. https://doi.org/10.1017/CBO9781107050990.014

[9] Gudder, S.P. (1988) Quantum Probability. Academic Press, San Diego.

[10] Freeze, K., Brown, M. and Kinney, W. (2012) The Phantom Bounce, a New Proposal for an Oscillating Cosmology. In: Mercini-Houghton, L. and Vaas, R., Eds., The Arrows of Time, a Debate in Cosmology. Fundamental Theories in Physics, Volume 172, Springer Verlag, Heidelberg, 149-156.

https://doi.org/10.1007/978-3-642-23259-6_7

[11] Novello, M. (2005) The Mass of the Graviton and the Cosmological Constant Puzzle. https://arxiv.org/abs/astro-ph/0504505

[12] Beckwith, A. (2021) A Solution of the Cosmological Constant, Using Multiverse Version of Penrose CCC Cosmology, and Enhanced Quantization Compared. https://doi.org/10.20944/preprints202102.0208.v1

[13] Beckwith, A. (2021) A Solution of the Cosmological Constant and DE Using Breakup of Primordial Black Holes, via a Criteria Brought Up by Dr. Freeze Which Initiates DE as Linked to Inflation. https://doi.org/10.20944/preprints202104.0210.v1 
[14] Sarkar, U. (2008) Particle and Astroparticle Physics. Taylor \& Francis Group, New York.

[15] Park, D., Kim, H. and Tamarayan, S. (2002) Nonvanishing Cosmological Constant of Flat Universe in Brane-World Scenario. Physics Letters B, 535, 5-10. https://doi.org/10.1016/S0370-2693(02)01729-X

[16] Ng, Y.J. (2007) Holographic Foam, Dark Energy and Infinite Statistics. Physics Letters B, 657, 10-14. https://doi.org/10.1016/j.physletb.2007.09.052

[17] Rosen, N. and Israelit, M. (1991) A Simple Model of the Universe without Singularities. In: Zichichi, A., de Sabbata, V. and Sánchez, N., Eds., Gravitation and Modern Cosmology, Ettore Majorana International Science Series, Vol. 56, Springer, Boston, 151-156. https://doi.org/10.1007/978-1-4899-0620-5_14

[18] Li, M., Li, X.-D., Wang, S. and Wang, Y. (2015) Dark Energy. Peking University Press, World Scientific, Singapore.

[19] t'Hooft, G. (2018) Time, the Arrow of time and Quantum Mechanics. Frontiers in Physics, 6, 81. https://doi.org/10.3389/fphy.2018.00081

[20] Beckwith, A.W. (2018) Initial Conditions for Defining an Arrow of Time at the Start of Inflation? Journal of High Energy Physics, Gravitation and Cosmology, 4, 787-795. https://doi.org/10.4236/jhepgc.2018.44044

[21] Kolb, E. and Turner, S. (1994) The Early Universe. Westview Press, Chicago.

[22] Dowker, F. (2005) Causal Sets and the Deep Structure of Space-Time. https://arxiv.org/abs/gr-qc/0508109

[23] Winitzki, S. (2009) Eternal Inflation. World Scientific Publishing Co., Singapore. https://doi.org/10.1142/6923

[24] Rosen, N. (1993) Quantum Mechanics of a Miniuniverse. International Journal of Theoretical Physics, 32, 1435-1440. https://doi.org/10.1007/BF00675204

[25] Wang, Q.D., Zhu, Z. and Unruh, W.G. (2017) How the Huge Energy of Quantum Vacuum Gravitates to Drive the Slow Accelerating Expansion of the Universe. Physical Review D, 95, Article ID: 103504. https://doi.org/10.1103/PhysRevD.95.103504

[26] Turner, M. (1983) The Origins of Density Fluctuations in the "New Inflationary Universe”. In: Gibbons, G.W., Hawking, S.W. and Siklos, S.T.C., Eds., The Very Early Universe, Cambridge University Press, New York, 297-309.

[27] Nakayama, K. and Tang, Y. (2019) Stochastic Gravitational Waves from Particle Origin. Physics Letters B, 788, 341-346. https://arxiv.org/pdf/1810.04975.pdf

[28] Remmen, G.N. and Carroll, S.M. (2014) How Many e-Folds Should We Expect from High-Scale Inflation? https://arxiv.org/pdf/1405.5538.pdf

[29] Bousso, R. and Hawking, S.W. (1996) Pair Creation of Black Holes during Inflation. https://arxiv.org/pdf/gr-qc/9606052.pdf

[30] Kiefer, C. (2003) On the Production of Primordial Black Holes in Inflationary Cosmology. https://www.mpifr-bonn.mpg.de/1360968/3rd_WG1_Kiefer.pdf

[31] Baker Jr., R. (2020) A Theory of Our Universe. Journal of High Energy Physics, Gravitation and Cosmology, 6, 609-622. https://doi.org/10.4236/jhepgc.2020.64041

[32] Calmet, X., Carr, B. and Winstanley, E. (2014) Quantum Black Holes. Springer Verlag, New York. https://doi.org/10.1007/978-3-642-38939-9

[33] Alves, M.E.S. Miranda, O.D. and Araujo, J.C.N. (2009) Can Massive Gravitons Be an Alternative to Dark Energy? Physics Letters B, 700, 283-288. https://arxiv.org/abs/0907.5190 
[34] Maggiore, M. (2007) Gravitational Waves, Volume 1, Theory and Experiment. Oxford University Press, Oxford.

[35] Kahniashvili, T., Brandenburg, A., Gogoberidze, G., Mandal, S. and Pol, A.R. (2021) Circular Polarization of Gravitational Waves from Early-Universe Helical Turbulence. Physical Review Research, 3, Article ID: 013193.

https://journals.aps.org/prresearch/abstract/10.1103/PhysRevResearch.3.013193 https://doi.org/10.1103/PhysRevResearch.3.013193

[36] Padmaabhan, T. (2016) Quantum Field Theory, the Why, What and How. Graduate Text in Physics, Springer Verlag, New York.

[37] Lightman, A., Press, W., Price, R. and Teukolsky, S. (1975) Problem Book in Relativity and Gravitation. Princeton University Press, Princeton. 